\title{
Adaptive evolution of the osmoregulation-related genes in cetaceans during secondary aquatic adaptation
}

\author{
Shixia Xu, Yunxia Yang, Xuming Zhou, Junxiao Xu, Kaiya Zhou and Guang Yang*
}

\begin{abstract}
Background: Osmoregulation was a primary challenge for cetaceans during the evolutionary transition from a terrestrial to a mainly hyperosmotic environment. Several physiological mechanisms have been suggested to maintain the water and salt balance in cetaceans, but their genetic and evolutionary bases remain poorly explored. The current study investigated the genes involved in osmoregulation in cetaceans and compared them with their counterparts in terrestrial mammals to test whether adaptive evolution occurred during secondary aquatic adaptation.
\end{abstract}

Results: The present study analyzed the molecular evolution of 11 osmoregulation-related genes in 11 cetacean species, which represented all of the major cetacean clades. The results demonstrated positive selection acting on angiotensin converting enzyme (ACE), angiotensinogen (AGT), SLC14A2, and aquaporin 2 (AQP2). This evidence for the positive selection of AQP2 and SLC14A2 suggests that the adaptive evolution of these genes has helped to enhance the capacity for water and urea transport, thereby leading to the concentration of urine, which is an efficient mechanism for maintaining the water balance. By contrast, a series of positively selected amino acid residues identified in the ACE and AGT (two key members of the renin-angiotensin-aldosterone system, RAAS) proteins of cetaceans suggests that RAAS might have been adapted to maintain the water and salt balance in response to a hyperosmotic environment. Radical amino acid changes in positively selected sites were distributed among most internal and terminal branches of the cetacean phylogeny, which suggests the pervasively adaptive evolution of osmoregulation since the origin of cetaceans and their subsequent diversification.

Conclusions: This is the first comprehensive analysis of the molecular evolution of osmoregulation-related genes in cetaceans in response to selection pressure from a generally hyperosmotic environment. Four genes, i.e., AQP2, SLC14A2, ACE, and AGT were subject to positive selection in cetaceans, which suggests that cetaceans may have adapted to maintain their water and salt balance. This also suggests that cetaceans may have evolved an effective and complex mechanism for osmoregulation.

Keywords: Adaptive evolution, Cetaceans, Osmoregulation, Positive selection

\section{Background}

Cetaceans are a highly specialized order of mammals that "returned" from the land to the sea approximately 53-56 million years ago $(\mathrm{Ma})$ and they ultimately became a successful and highly diverse group of fully aquatic mammals [1]. During this evolutionary transition from land to generally high salt waters, the maintenance of homeostasis via

\footnotetext{
* Correspondence: gyang@njnu.edu.cn

Jiangsu Key Laboratory for Biodiversity and Biotechnology, College of Life Sciences, Nanjing Normal University, 1 Wenyuan Road, Nanjing 210023, China
}

\section{Biomed Central}

(c) 2013 Xu et al.; licensee BioMed Central Ltd. This is an Open Access article distributed under the terms of the Creative Commons Attribution License (http://creativecommons.org/licenses/by/2.0), which permits unrestricted use, distribution, and reproduction in any medium, provided the original work is properly cited. the regulation of water and electrolyte levels was one of the most critical challenges they encountered.

Marine mammal osmoregulation has been investigated for over a century, but it is still not clear how they developed mechanisms to conserve fresh water and to avoid dehydration. The acquisition of fresh water by cetaceans may occur via seawater consumption, water from food, and water derived from metabolism [2,3]. Compared with terrestrial mammals, marine mammals such as cetaceans consume a water-rich diet of fishes and marine invertebrates [2]. Thus, the water found in their prey 
and the water derived subsequently from vigorous metabolism are considered to be their primary sources of fresh water [2-4]. In addition, recent studies present evidence that cetaceans acquire fresh water from sea-water because they excrete higher $\mathrm{Na}^{+}$levels than those found in sea-water, especially during fasting periods [5-7]. Moreover, physiological studies show that the water conservation capacity of cetaceans may be better than that of their terrestrial relatives $[8,9]$.

Another primary osmotic challenge for cetaceans is the excretion of the excess salt $\left(\mathrm{Na}^{+}\right)$ingested with their prey and sea-water. Anatomical evidence suggests that the 'specialized' kidneys of cetaceans might be important for salt excretion and water conservation $[4,10]$. Cetacean kidneys are relatively large compared with those of other mammals and they contain many small, independently functional renules $[11,12]$. Each renule is similar to those found in other mammals $[12,13]$. In addition, the relatively large ratio of medulla to renal cortex in cetaceans [11] allows them to produce highly concentrated urine because of enhanced water reabsorption [12]. Thus, the reniculate kidney and increased medullary thickness may be a highly efficient system for salt excretion and water conservation, which represents an evolutionary response to a hyperosmotic environment [14-16].

The hormonal regulation of the salt and water balance is another efficient mechanism in marine mammals, which is also present in terrestrial mammals (reviewed in [3]). Five primary hormones, i.e., angiotensin (ANG, including ANG I, II, or III), renin, aldosterone, atrial natriuretic peptide (ANP), and vasopressin (AVP), are responsible for regulating the salt and water balance in the kidneys of mammals [4]. Three of these, i.e., renin (REN), ANG, and aldosterone, operate via the reninangiotensin-aldosterone system (RAAS) to modulate the activity of $\mathrm{Na}^{+}-\mathrm{K}^{+}$-ATPase and to increase water reabsorption by mammals $[17,18]$. RAAS is known to be present in cetaceans and it appears to regulate the salt balance $[4,19]$. ANP is also responsible for regulating the salt balance by inhibiting $\mathrm{Na}^{+}$transport in the inner medullary collecting ducts [20], although this has not been bioassayed in cetaceans. AVP also contributes significantly to water retention by increasing water reabsorption in the renal collecting ducts [21], whereas large increases in water reabsorption rely heavily on AVPregulated aquaporins (AQPs), which are responsible for water transport [22,23]. In cetaceans, however, an early study detected no association between the urine flow rate and the plasma AVP concentration in fasting dolphins [24].

Several physiological adaptations for water conservation and salt excretion have been suggested in cetaceans that may have facilitated their adaptation to a high salt environment, but the molecular bases that underlie these mechanisms remain poorly explored. Many genes have been identified that are involved with osmoregulation. For example, $\mathrm{Na}^{+}-\mathrm{K}^{+}$-ATPase, an important salt regulating system, is crucial for maintaining intracellular homeostasis but it also provides the driving force for ion transport in a variety of osmoregulatory epithelia [25]. $\mathrm{Na}^{+}-\mathrm{K}^{+}$-ATPase is a heterodimer with a catalytic $\alpha-$ subunit and a glycosylated $\beta$-subunit [26]. The $\alpha 1$ subunit, one of four isoforms ( $\alpha 1-\alpha 4)$, may have a housekeeping role in maintaining the osmotic balance and in regulating the cell-volume [27]. AQPs are important membrane channels that regulate the water balance in the body [28,29]. In particular, some AQPs, such as $\mathrm{AQP} 1-\mathrm{AQP} 4$, are specialized for water re-absorption in the kidney, which has an indispensable role in urine concentration [30]. The increased water re-absorption due to AQPs is heavily reliant on AVP stimulation [21]. The urea transporters (UTs) that are highly expressed in the mammalian kidney may also be effective mechanisms for urine concentration [31]. Two genes (SLC14A2 and SLC14A1) encode urea transporters in the mammalian kidney (NCBI http://www.ncbi.nlm.niv. gov). Thus, the renal UTs in mammals act together with the renal AQPs and have integral roles in regulating the body water balance [31]. The present study investigated the coding regions of the $\mathrm{Na}^{+}-\mathrm{K}^{+}$-ATPase $\alpha 1$ subunit, AQP 1-4, SLC14A2, SLC14A1, RAAS (including REN, AGT, and ACE), ANP, and AVP genes in representative cetacean lineages. The genes of cetaceans were compared with those of terrestrial mammals to determine whether they had evolved adaptively during the cetacean transition from the land to the sea, and during their subsequent radiation into waters throughout the world.

\section{Results}

The 10 osmoregulation-related genes examined in this study were sequenced successfully in 11 cetacean species. All genes were intact and there were no premature stop codons or frame-shift mutations, which indicated the presence of functional proteins in cetaceans. Unfortunately, the AVP gene was not amplified successfully in all 11 cetacean species, although various experimental optimizations were attempted. Thus, only the cetacean AVP sequence derived from the baiji (Lipotes vexillifer) genome (data unpublished) was used to test selection in the following analysis.

\section{Molecular evolution of osmoregulatory genes in cetaceans}

A pair of site models (M8a vs M8) [32,33] was used to test whether specific codons in the osmoregulatory genes had been subjected to positive selection. Likelihood ratio tests (LRTs) showed that the model incorporating selection 
(M8) fitted significantly better than the neutral model (M8a) for ACE, REN, and SLC14A2 (Table 1), whereas no significant evidence of positive selection was found for the other eight genes. In model 8 , the most stringent model implemented in PAML, a small proportion of codons $(0.968 \%, 1.720 \%$, and $2.207 \%)$ were estimated to be under selection, with $\omega$ values of $2.077,16.110$, and 1.529 for the ACE, REN, and SLC14A2 genes, respectively. In addition, six, four, and eight sites were identified by the BEB approach as having posterior probabilities $\geq 0.80$.

Further tests were made to determine whether a similar selection pattern also occurred in cetaceans. ACE

Table 1 CODEML analysis of osmoregulatory genes (11 genes) and evidence of positive selection on the ACE, AGT, SLC14A2, REN, AQP2, and AVP genes in mammals and cetaceans

\begin{tabular}{|c|c|c|c|c|c|}
\hline Genes & Models & $-\ln L$ & $2 \Delta \operatorname{LnL}$ & $P$ value ${ }^{a}$ & $\omega$ values \\
\hline ACE & Site model & & & & \\
\hline \multirow[t]{11}{*}{ (1033aa) } & \multicolumn{5}{|c|}{ Dataset I: All mammals (28 sequences) } \\
\hline & M8a & 20856.619 & & & $\omega=1$ \\
\hline & M8 & 20853.691 & 5.856 & 0.016 & $\omega=2.077$ \\
\hline & \multicolumn{5}{|c|}{ Dataset II: cetaceans (11 sequences) } \\
\hline & M8a & 5910.391 & & & $\omega=1$ \\
\hline & M8 & 5905.151 & 10.480 & 0.001 & $\omega=4.855$ \\
\hline & \multicolumn{5}{|c|}{ Branch site model } \\
\hline & \multicolumn{5}{|c|}{ Dataset I: All mammals (28 sequences) } \\
\hline & \multicolumn{5}{|c|}{ Branch n (terminal branch of Grampus griseus) } \\
\hline & Null & 21017.590 & & & $\omega 0=0.070, \omega 1=1, \omega 2=1$ \\
\hline & Alternative & 21010.004 & 15.172 & 0.002 & $\omega 0=0.070, \omega 1=1, \omega 2=258.836$ \\
\hline AGT & Site model & & & & \\
\hline \multirow[t]{8}{*}{ (324aa) } & \multicolumn{5}{|c|}{ Dataset II: cetaceans (10 sequences) } \\
\hline & M8a & 2017.713 & & & $\omega=1$ \\
\hline & M8 & 2014.489 & 6.448 & 0.011 & $\omega=4.134$ \\
\hline & \multicolumn{5}{|c|}{ Branch site model } \\
\hline & \multicolumn{5}{|c|}{ Dataset I: All mammals (30 sequences) } \\
\hline & \multicolumn{5}{|c|}{ Branch n (terminal branch of G. griseus) } \\
\hline & Null & 10615.771 & & & $\omega 0=0.154, \omega 1=1, \omega 2=1$ \\
\hline & Alternative & 10601.717 & 28.107 & $<0.001$ & $\omega 0=0.154, \omega 1=1, \omega 2=952.14$ \\
\hline
\end{tabular}

\begin{tabular}{|c|c|c|c|c|c|}
\hline \multirow{2}{*}{$\begin{array}{l}\text { SLC14A2 } \\
\text { (861aa) }\end{array}$} & \multicolumn{5}{|l|}{ Site model } \\
\hline & \multicolumn{5}{|c|}{ Dataset I: All mammals (28 sequences) } \\
\hline & M8a & 15199.892 & & & $\omega=1$ \\
\hline & M8 & 15197.706 & 4.372 & 0.037 & $\omega=1.529$ \\
\hline & \multicolumn{5}{|c|}{ Dataset II: cetaceans (10 sequences) } \\
\hline & M8a & 4602.652 & & & $\omega=1$ \\
\hline & M8 & 4597.947 & 9.410 & 0.002 & $\omega=2$ \\
\hline REN & \multicolumn{5}{|l|}{ Site model } \\
\hline \multirow[t]{3}{*}{ (407aa) } & \multicolumn{5}{|c|}{ Dataset I: All mammals (25 sequences) } \\
\hline & M8a & 7542.944 & & & $\omega=1$ \\
\hline & M8 & 7531.661 & 22.565 & $<0.001$ & $\omega=16.110$ \\
\hline AQP2 & \multicolumn{5}{|c|}{ Branch site model } \\
\hline \multirow[t]{4}{*}{ (202aa) } & \multicolumn{5}{|c|}{ Dataset I: All mammals (27 sequences) } \\
\hline & \multicolumn{5}{|c|}{ Branch c (ancestral toothed whales) } \\
\hline & Null & 3827.416 & & & $\omega 0=0.037, \omega 1=1, \omega 2=1$ \\
\hline & Alternative & 3822.989 & 8.854 & 0.074 & $\omega 0=0.038, \omega 1=1, \omega 2=999$ \\
\hline
\end{tabular}


and SLC14A2 were found to be under positive selection in the cetacean dataset where the LRTs of the site model were statistically significant (ACE: M8a vs M8: $P=0.022$; SLC14A2: M8a vs M8: $P=0.002)$; however, unlike the dataset containing all mammals, REN did not show any sign of positive selection (M8a vs M8: $P=0.114$ ). By contrast, positive selection was detected in the AGT gene (M8a vs M8: $P=0.011)$. In the M8 model, the average $\omega$ values of cetaceans were $4.855,4.134$, and 2.0 for ACE, AGT, and SLC14A2, respectively, and a small proportion of codons (ACE: $1.936 \%$ or 20 codons; AGT: $1.852 \%$ or six codons; and SLC14A2: $3.136 \%$ or 27 codons) were identified as being under positive selection (Table 1). Nine, four, and 19 codons were identified as being positively selected using the $\mathrm{BEB}$ approach with posterior probabilities $\geq 0.8$ in ACE, AGT, and SLC14A2, respectively.

The branch-site model was then used to test for positive selection in individual codons in each lineage of cetaceans (a-u in Figure 1) and the lineages of other groups such as cetartiodactyls, carnivores, rodents, and primates across the phylogeny of all mammals (Additional file 1: Figure S1). The LRT tests showed that there was evidence of positive selection in one cetacean-specific lineage (test 2 , $P<0.05)$ after correcting for multiple testing, i.e., the terminal branch of Grampus griseus for both ACE $(P=0.002)$ and AGT $(P<0.001)$ (Table 1 and Figure 1$)$. Almost significant positive selection was found at branch $\mathrm{b}$, which led to the last common ancestor of odontocetes for AQP2 $(P=0.074)$ after corrections. Fourteen codons were under positive selection in the two cetacean-specific lineages (Figure 1, Additional file 2: Table S1), whereas no significant signs of positive selection were detected in the lineages of the other groups, i.e., cetartiodactyls, carnivores, rodents, and primates.

Furthermore, 12 positively selective sites were identified using the random effects likelihood (REL) method. Of these putative positively selected sites, 12 (ACE: 158, 246, 373, 315, 792, 808, 836, 911; AGT: 85, 111, 219; AQP2: 105) were detected by both methods whereas only one codon (AGT: 111) was detected by three methods. Thus, four genes (ACE, AGT, SLC14A2, and AQP2) and 45 codons (18 in ACE, five in AGT, 19 in SLC14A2, and three in AQP2) were shown to have been positively selected in cetaceans (Additional file 2: Table S1). The radical amino acid changes in the 45 positively selected codons were scattered throughout most of the cetacean phylogeny (Figure 1). Furthermore, the overwhelming majority of these changes were radical (exact binomial test: $P<0.001$; Additional file 2: Table S1), which may be additional evidence for positive selection [34-36].

\section{Spatial distribution of the positively selected sites in the 3D structures}

The functional significance of the positively selected sites was investigated further by locating them in the 3D structures of their corresponding genes, which showed that most of the positively selected sites were localized in, or close to, the functional region in the predicted 3D structures of ACE and SLC14A2 (Additional file 3: Figure S2). In the $\mathrm{ACE}$ gene, all the 18 positively selected sites were localized in the two zinc-dependent catalytic domains, i.e., $72.222 \%(13 / 18)$ and $27.778 \%(5 / 18)$ in the $\mathrm{N}$ and $\mathrm{C}$ domains, respectively (Additional file 3: Figure S2). For the SLC14A2 gene, one positively selected site that had undergone radical changes was located in the protein

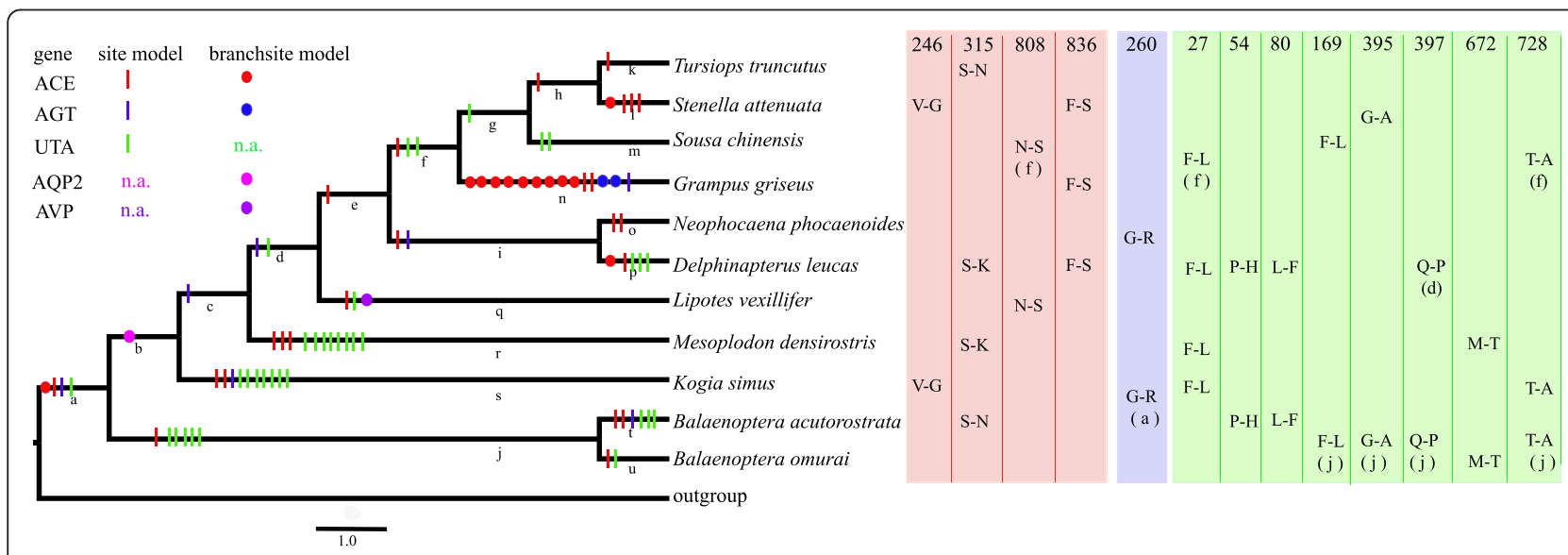

Figure 1 Radical amino acid changes in selected sites across the cetacean phylogeny. Parallel amino acid substitutions occurred in three selected genes (ACE, AGT, and SLC14A2) shown on the right of the figure. The selected genes and their corresponding parallel amino acid substitutions in cetaceans are marked with different colors, i.e., ACE (red), AGT (blue), SLC14A2 (green), and AQP2 (purple). Selected sites identified using site models and branch-site models are indicated separately by vertical lines and circles, respectively. For each amino acid position, the parallel amino acid is labeled on the right of the corresponding terminal branches, while $a, d$, and $f$ in parentheses indicate the internal branches where the parallel changes occurred. Terrestrial mammals were used as outgroups including cetartiodactyls, carnivores, rodents, and primates. 
kinase C (PKC) consensus phosphorylation site (Ser-490) in cetaceans.

\section{Discussion}

Pervasively adaptive evolution of cetacean osmoregulation Osmoregulation has been studied in marine mammals for over 100 years but there are still no clear conclusions about how their osmoregulation capacity was enhanced in response to a hyperosmotic environment. Most arguments are focused on whether marine mammals can drink sea-water and whether marine mammals can excrete salt-concentrated urine. It is likely that whales ingest sea-water passively while preying on marine animals. In particular, baleen whales filter invertebrates through their baleen plates that are isotonic with seawater so it is inevitable that they will ingest the seawater in the body fluids of their prey [37]. Furthermore, the ingestion of seawater by cetaceans was confirmed by a recent observation that the $\mathrm{Na}^{+}$concentration of urine is higher than that of sea-water after consuming fish and sea-water [7]. There is still some controversy over whether cetaceans can excrete salt-concentrated urine, but recent studies provide evidence that cetaceans, which are equipped with highly efficient renicules, can produce more concentrated urine than their close terrestrial relatives by concentrating $\mathrm{Na}^{+}$and urea $[4,9,38]$. By contrast, previous studies have questioned the ability of cetaceans to concentrate urine $[39,40]$.

Most previous studies have focused on the unique renal physiology of marine mammals (reviewed in [3]). Several genes involved with osmoregulation, such as UTs, AQP1, and AQP2, have been cloned in one or two species [40-43], but there is still no comprehensive understanding of the evolution of the osmoregulationrelated genes in cetaceans. Thus, the present study conducted comparative analyses of the selective pressure on osmoregulatory genes in cetaceans and terrestrial mammals to test whether adaptive evolution occurred during the origin and diversification of cetaceans. Four lines of evidence support the hypothesis that significantly adaptive molecular evolution of cetacean osmoregulation has occurred. First, the selection analysis showed that four genes involved with osmoregulation, i.e., ACE, AGT, $\mathrm{AQP} 2$, and SLC14A2, were subjected to strong positive selection in cetacean-specific lineages, whereas no selection was observed in terrestrial mammalian lineages such as cetartiodactyls, carnivores, rodents, and primates. Second, adaptive evolution was further supported by evidence that radical changes in amino acids have occurred significantly more often than conservative changes in cetacean osmoregulation-related genes. Third, the positively selected sites were localized in, or close to, the functional regions in the predicted $3 \mathrm{D}$ structures of the $\mathrm{ACE}$ and SLC14A2 genes. Finally, 13 codons (ACE: 246, 315,
808, and 836; AGT: 260; SLC14A2: 27, 54, 80,169, 395, 397, 672, and 728) exhibited parallel amino acid changes in different cetacean lineages (Figure 1). Overall, the significantly positive selection identified in cetaceans suggests an enhancement of their osmoregulation capacity when they shifted their habitat from the land to the sea.

Remarkably, radical changes in amino acids occurred at positively selected sites scattered throughout the entire cetacean phylogeny, from the most common ancestral branch of cetaceans to the terminal branches. This suggests the pervasively adaptive evolution of cetacean osmoregulation. Osmoregulation is a basic requirement of cetaceans that maintains homeostasis in an environment with a high salt concentration so any failure of osmoregulation may lead to an imbalance of water or salt, which may cause further maladaptations in an aquatic environment, possibly causing the death of animals. Thus, it is reasonable to assume that different cetaceans require a fine osmoregulation capacity which has driven the relevant genes to evolve adaptively in response to continuous changes in their osmotic environments since their origin and subsequent diversification in waters throughout the world.

During cetacean evolution, the diversification of river dolphins and their dispersal from sea water to fresh water is a distinct transition. In contrast to their marine counterparts, river dolphins generally inhabit a low-salt (hypoosmotic) habitat that imposes a low osmoregulatory pressure on dolphins because they no longer experience problems conserving fresh water. However, signs of significantly positive selection in ACE, AGT, and SLC14A2 were unexpectedly identified in the baiji lineage, which was the only river dolphin examined in this study. This might be explained by the large difference between the freshwater habitat of river dolphins and that of oceanic dolphins in the sea. This major habitat change presented a new and different osmotic challenge to river dolphins, i.e., maintaining their electrolyte balance, which caused them to modify their osmoregulatory system further as an evolutionary response. To some degree, this may be supported indirectly by the anatomical and physiological differences between freshwater and marine finless porpoises [44]

\section{Enhanced water, salt, and urea regulation during cetacean osmoregulation}

The positive selection on SLC14A2 and AQP2 in the cetaceans considered in the present study supports a previous report that whales can produce higher concentrations of urea in their urine than terrestrial mammals [9]. AQP2 is localized in the collecting tubules/ducts of cetacean renicules, which is also the case in terrestrial mammals, and this protein is essential for the AVP-dependent reabsorption of water $[43,45,46]$. The positive selection in 
AQP2 in the cetaceans in the present study suggests an enhanced capacity for water reabsorption in the renal collecting ducts of cetaceans, which could further improve the ability to concentrate salt and urea in the urine. It should be noted that SLC14 A2 knockout mice have a major urinary concentration defect [47], which suggests SLC14A2 plays an important role in maintaining a high concentration of urea. The 19 positively selected sites identified in SLC14A2 in cetaceans also suggest that SLC14A2 must have been adapted to produce high concentration urine in response to a hyperosmotic environment. Furthermore, the positively selected site (490) in the PKC consensus phosphorylation sequence, which caused a radical amino acid change from a polar serine to a nonpolar isoleucine, suggests that whales can concentrate urine by activating PKC and increasing the permeability of urea.

RAAS is responsible for the water and salt balance in mammals [4]. The AGT and ACE genes, which are key members of RAAS, were under positive selection across cetaceans, suggesting cetaceans might have evolved a more effective RAAS to maintain osmoregulation. ANG II is a very powerful regulator of aldosterone release via the adrenal gland in RAAS, which in turn promotes sodium and water reabsorption in the kidneys. ANG II is synthesized via the cleavage of AGT in kidneys, which is mediated by the proteolytic enzyme REN to form ANG I, and this is rapidly converted by ACE to ANG II. Thus, ACE and AGT may be highly important for controlling the formation of ANG II, stimulating aldosterone release, and further regulation of the water and salt balance [18]. This is consistent with previous physiological studies where the plasma concentrations of aldosterone in most cetaceans were higher than those in camels and cattle [9]. By contrast, $\mathrm{Na}^{+}-\mathrm{K}^{+}$-ATPase is a key enzyme involved with direct $\mathrm{Na}^{+} / \mathrm{K}^{+}$transport, although it is found in many other tissues and is not exclusive to the kidneys. Therefore, no signs of positive selection were detected to indicate significant constraints on this gene, which was probably because of the basic requirement for its function in all tissues. Thus, functional studies of these osmoregulation-related genes are necessary in cetaceans in the future.

\section{Is widespread positive selection in cetaceans due to the effective population size $(\mathrm{Ne})$ ?}

Population genetic theory predicts that the efficiency of selection should decrease as the effective population size decreses [48]. Thus, selection is relatively ineffective compared with genetic drift and mutation in species with a low $N e$, and vice versa [49]. In addition, the substitution process is dictated mainly by mutation in species with a low $N e$. This means that we can expect a higher value of $\omega$ with a low population size compared with a high population size. However, three lines of evidence did not support this prediction in the present study. First, the average $\omega$ value $\left(\omega=0.22, d_{\mathrm{N}} / d_{\mathrm{S}}=0.012 /\right.$ 0.056) of the full-length protein in the common cetacean lineage (including the bottlenose dolphin Tursiops truncatus and the baiji $L$. vexillifer) was comparable to that in the mouse (Mus musculus; $\omega=0.14, d_{\mathrm{N}} / d_{\mathrm{S}}=$ $0.054 / 0.39$ ) (unpublished data), considering that smaller mammals tend to have larger effective population sizes [50]. Second, not all cetacean species have a low Ne. For example, the effective population size of Pacific gray whales (Eschrichtius robustus) has been estimated at between 31,175 and 38,084 breeding adults [51]. Finally, osmoregulation plays a crucial role in cetacean life so even a few mutations could be fatal. Thus, the fixation of mutations in the osmoregulation-related genes of cetaceans must be attributed to selection, which is fairly independent of the effective population size. Thus, all of fixed mutations in cetaceans would increase the overall fitness and adaptive response to hyperosmotic environments.

\section{Conclusions}

The osmoregulation of marine mammals has been investigated for over a century, but its genetic basis remains poorly explored. The present study is one of the first comprehensive analyses of the genetic basis of cetacean osmoregulation. This study detected positive selection particularly in the AQP2, SLC14A2, ACE, and AGT genes of cetaceans, which suggests that cetaceans may have adapted to maintain their water and salt balance in response to a mainly hyperosmotic environment. This also suggests that cetaceans may have evolved an effective and complex mechanism for osmoregulation.

\section{Methods}

\section{Sequence acquisition and compilation}

Eleven cetacean species were sampled, two mysticetes and nine odontocetes (Additional file 4: Table S2). All the cetacean samples used in our study were collected from dead individuals in the wild so that no ethics statement is required. Voucher specimens are preserved at Jiangsu Key Laboratory for Biodiversity and Biotechnology, College of Life Sciences, Nanjing Normal University (NNU), China. The exons of each osmoregulatory gene were sequenced and concatenated, before being analyzed together. Gene sequences from other mammals were downloaded from the OrthoMaM, which is a database that contains orthologous genomic markers for mammals [52]. Only high quality and high integrity sequences were used (the accession numbers for each gene are shown in Additional file 4: Table S2). A total of 47 mammalian species included in 16 orders were analyzed in the present study. The nucleotide and deduced amino acid sequences of each gene were aligned using CLUSTALX 1.83 [53] and MEGA 5.0 [54]. The aligned 
sequences and phylogenetic trees were deposited in TreeBase (http://purl.org/phylo/treebase/phylows/study/ TB2:S14426).

\section{Selective pressure detection}

Selective pressure was tested based on the mammalian phylogeny by comparing the nonsynonymous/synonymous substitution ratios $\left(\omega=d_{\mathrm{N}} / d_{\mathrm{S}}\right)$ with $\omega=1,<1$, and $>1$, which indicated neutral evolution, purifying selection, and positive selection, respectively. The $\omega$ ratios were estimated using the codon-based maximum likelihood models implemented in the CODEML program in PAML 4.4 [55]. The well-supported phylogeny of Laurasiatheria [56] and primates [57] was used as the input tree in all analyses (Additional file 1: Figure S1). The phylogenetic relationships were also reconstructed using Bayesian inference (BI) for each gene. The resulting trees were similar to the above well-supported phylogeny, with some minor differences in a few branches. Moreover, only the latter analysis is reported because selective detection using the gene tree produced almost identical results to those obtained using the well-supported species phylogeny.

To identify the probabilities of sites under positive selection in each gene, site models were implemented where $\omega$ could vary among sites $[58,59]$, which facilitated the analysis of datasets including all mammals or cetaceans only. In particular, a pair of models was tested, i.e., M8a (almost neutral; beta distribution: $0<\omega_{0}<1$ and $\left.\omega_{1}=1\right)$ vs M8 (positive selection; beta distribution: $0<\omega_{0}<1$ and $\omega_{1}>1$ ) $[60,61]$. Positive selection often operates episodically on a few amino acid sites in a small number of lineages in a phylogenetic tree [62]. Thus, branch-site models were considered in this study that allow $\omega$ to vary among sites in the protein and across branches on the tree. Modified branch-site model A (test 2) was performed for each gene in each cetacean lineage across the phylogeny of all mammals. All of the positively selective sites were identified using a Bayes Empirical Bayes (BEB) analysis [55] with posterior probabilities $\geq 0.80$. Likelihood ratio test $\mathrm{p}$-values were adjusted for multiple testing with a Benjamini \& Hochberg's procedure $[63,64]$ and threshold of 0.05 . In addition, positively selected sites was also inferred using the Random effects likelihood (REL) method implemented in the DATAMONKEY web server [65]. Bayes factor $>50$ for REL was implemented. The DATAMONKEY has the advantage that they can improve the estimation of the $d_{\mathrm{N}} / d_{\mathrm{S}}$ ratio by incorporating variation in the rate of synonymous substitution [65].

The radical amino acid changes that occurred in the positively selected sites were also counted and mapped onto each branch in the cetacean phylogeny. Ancestral sequences for all interior nodes were inferred based on empirical Bayesian methods implemented in the CODEML program in the PAML 4.4 package [55]. Conservative or radical nonsynonymous substitutions were determined according to the methods proposed by Zhang [66].

\section{Mapping of positively selected sites onto 3D structures}

The 3D structures of genes identified as being under positive selection were predicted following the homology modelling using the SWISS-MODEL (http://swissmodel.expasy. org) [67]. The protein sequences of positively selected genes were derived from the Tursiops truncutus genome, which were obtained using ENSEMBL (http://www.ensembl.org/ index.html). To provide further insights into the functional significance of these positively selected sites, they were mapped onto the $3 \mathrm{D}$ structure using PYMOL (http://pymol.sourceforge.net/).

\section{Additional files}

Additional file 1: Figure S1. A well supported phylogeny of mammals used for selective pressure analysis in PAML. Tree topologies of

Laurasiatheria and primates were from Zhou et al. [56] and Perelman et al. [57], respectively. Different orders of mammals were marked with different colors. Branches a- $u$ in the tree included in cetaceans are used in the branch-site models tests, and results listed in Table 1 and Figure 1.

Additional file 2: Table S1. Radical or conservative changes occurred at positively selected sites detected in cetaceans using the site models and branch-site models.

Additional file 3: Figure S2. Spatial distribution of positively selected sites in the three-dimensional (3D) structure of cetacean ACE (A), AGT (B), AQP2 (C), SLC14A2 (D) genes. The 3D structure of ACE and UTA contains two (a1-ACE and a2-ACE) and three domains (d1-SLC14A2, d2- SLC14A2, d3- SLC14A2), respectively. All the positively selected sites were mapped onto 3D structure of each gene.

Additional file 4: Table S2. Sequence data used in this study, including taxonomy and accession numbers or Ensembl ID.

\section{Abbreviations}

RAAS: Renin-angiotensin-aldosterone system; ACE: Angiotensin converting enzyme; AGT: Angiotensinogen; ANG: Angiotensin; ANP: Atrial natriuretic peptide; AVP: Vasopressin; AQPs: Aquaporins; UTs: Urea transporters.

\section{Competing interests}

The authors declare that they have no competing interests.

\section{Authors' contributions}

SX and GY conceived the project and designed the experiments. $Y Y$ and $X Z$ performed the experiments, and SX, YY and JX analyzed the data. SX wrote the manuscript, and $K Z$ and $G Y$ improve the manuscript. All authors read and approved the final manuscript.

\section{Acknowledgements}

Authors wish to thank Mr Xinrong Xu for collecting the samples for many years. We thank Dr. Tong Shen for helpful discussion on the manuscript. This work was supported by the National Natural Science Foundation of China (NSFC) to GY (grant no. 31172069) and SXX (grant no. 31000953), the Priority Academic Program Development of Jiangsu Higher Education Institutions to GY and SXX, Specialized Research Fund for the Doctoral Program of Higher Education to SXX (grant no. 20103207120010) and GY (grant no. 20113207130001), and the Cultivation Plan for Excellent Doctorial Dissertations of Nanjing Normal University to YXY (grant no. CXLX12_0402).

Received: 18 March 2013 Accepted: 2 September 2013

Published: 9 September 2013 


\section{References}

1. Thewissen JGM, Cooper LN, Clementz MT, Bajpai S, Tiwari BN: Whales originated from aquatic artiodactyls in the Eocene epoch of India. Nature 2007, 450:1190-1194.

2. Costa DP: Osmoregulation. In Encyclopedia of marine mammals. Edited by Perrin WF, Thewissen JGM, Wursig B. San Diego: Academic Press; 2002:837-842.

3. Ortiz RM: Osmoregulation in marine mammals. J Exp Biol 2001, 204:1831-1844.

4. Ortiz RM, Wade CE, Ortiz CL: Prolonged fasting increases the response of the renin-angiotensin-aldosterone system, but not vasopressin levels, in post weaned northern elephant seal pups. Gen Comp Endocrinol 2001, 119:217-223.

5. Hui CA: Seawater consumption and water flux in the common dolphin Delphinus delphis. Physiol Zool 1981, 54:430-440.

6. Telfer NN, Cornell LHL, Prescott JHJ: Do dolphins drink water? J Am Vet Med Assoc 1970, 157:555-558

7. Ridgway S, Venn-Watson S: Effects of fresh and seawater ingestion on osmoregulation in Atlantic bottlenose dolphins (Tursiops truncatus). J Comp Physiol B, Biochem Syst Environ Physiol 2010, 180:563-576.

8. Vander AJ: Renal physiology. New York: McGraw Hill Inc; 1995.

9. Birukawa N, Ando H, Goto M, Kanda N, Pastene LA, Nakatsuji H, Hata H, Urano A: Plasma and urine levels of electrolytes, urea and steroid hormones involved in osmoregulation of cetaceans. Zool Sci 2005, 22:1245-1257.

10. Hedges NA, Gaskin DE, Smith GJD: Rencular morphology and renal vascular system of the harbour porpoise Phocoena phocoena (L.). Can J Zool 1979, 57:868-875.

11. Arvy $L$ : The kidney, renal parasites and renal secretion in cetaceans. In Investigations on Cetacea. Edited by Pilleri G. Berne: Der Bund; 1973:232-310.

12. Pfeiffer $C$ : Renal cellular and tissue specializations in the bottlenose dolphin (Tursiops truncatus) and beluga whale (Delphinapterus leucas). Aquat Mamm 1997, 23:75-84

13. Cave AJE, Aumonier FJ: Further observations on the cetacean reniculus. J R Microsc Soc 1965, 84:539-548.

14. Slijper EJ: Organ weights and symmetry problems in porpoises and seals. Arch Néerl Zool 1958, 13:97-113.

15. Kamiya T, Yamasaki F: Organ weights of Pontoporia blainvillei and Platanisto gangetica (Platanistidae). Sci Rep Whales Res Inst 1974, 26:265-270.

16. Maluf NS, Gassman JJ: Kidneys of the killer whale and significance of reniculism. Anat Rec 1998, 250:34-44

17. Sealey JE, Laragh $J \mathrm{H}$ : Renin-angiotensin-aldosterone system and the renal regulation of sodium, potassium, and pressure homeostasis. In Handbook of physiology-section 8: renal physiology. 2nd edition. Edited by Windhager EE. New York: Oxford University Press; 1992:1409-1541.

18. Harrison-Bernard LM: The renal renin-angiotensin system. Adv Physiol Educ 2009, 33:270-274

19. Malvin RL, Ridgway SH, Cornell L: Renin and aldosterone levels in dolphins and sea lions. Proc Soc Exp Biol Med 1978, 157:665-668

20. Levy $M$, Cernacek $P$ : Renal response to ANP in normal dogs during extreme inhibition of distal $\mathrm{Na}+$ transport. Kidney Int 1993, 43:1236-1242.

21. Caldwell HK, Young WS: Oxytocin and vasopressin: genetics and behavioral implications. In Handbook of neurochemistry and molecular neurobiology: neuroactive proteins and peptides. 3rd edition. Edited by Lajtha A, Lim R. Berlin: Springer; 2006:573-607.

22. Nielsen S, Chou CL, Marples D, Christensen El, Kishore BK, Knepper MA Vasopressin increases water permeability of kidney collecting duct by inducing translocation of aquaporin-CD water channels to plasma membrane. Proc Natl Acad Sci U S A 1995, 92(4):1013-1017.

23. Fenton RA, Moeller HB, Hoffert JD, Yu MJ, Nielsen S, Knepper MA: Acute regulation of aquaporin-2 phosphorylation at Ser-264 by vasopressin. Proc Natl Acad Sci U S A 2008, 105:3134-3139.

24. Malvin RL, Bonjour JP, Ridgway SH: Antidiuretic hormone levels in some cetaceans. Proc Soc Exp Biol Med 1971, 136:1203-1205.

25. Lingrel JB, Kuntzweiler T: $\mathrm{Na}^{+} / \mathrm{K}^{+}$-ATPase. J Biol Chem 1994, 269:19659-19662.

26. Sweadner KJ: Isozymes of the $\mathrm{Na}+/ \mathrm{K}+-\mathrm{ATPase}$. Biochim Biophys Acto 1989, 988:85-220.

27. Blanco G, Xie Z, Mercer RW: Functional expression of the a2 and a3 isoforms of the $\mathrm{Na}$, K-ATPase in baculovirus-infected insect cells. Proc Natl Acad Sci U S A 1993, 90:1824-1828.
28. Borghi L, Meschi T, Schianchi T, Briganti A, Guerra A, Allegri F, Novarini A: Urine volume: stone risk factor and preventative measure. I Nephrol 1999, 81:31-37.

29. Verkman AS, Mitra AK: Structure and function of aquaporin water channels. Am J Physiol 2000, 278:13-28.

30. Yamamoto T, Sasaki S: Aquaporins in the kidney: emerging new aspects. Kidney Int 1998, 54:1041-1051.

31. Smith CP: Mammalian urea transporters. Exp Physiol 2008, 94:180-185.

32. Swanson WJ, Nielsen R, Yang Q: Pervasive adaptive evolution in mammalian fertilization proteins. Mole Biol Evol 2003, 20:18-20.

33. Wong WSW, Yang Z, Goldman N, Nielsen R: Accuracy and power of statistical methods for detecting adaptive evolution in protein coding sequences and for identifying positively selected sites. Genetics 2004, 168:1041-1051.

34. Hughes AL: Coevolution of the vertebrate integrin alpha- and beta-chain genes. Mol Biol Evol 1992, 9:216-234.

35. Hughes AL: Evolution of cysteine proteinases of eukaryotes. Mol Phylogenet Evol 1994, 3:310-321

36. Hughes AL, Hughes MK: Adaptive evolution in the rat olfactory receptor gene family. J Mol Evol 1993, 36:249-254.

37. Ohsumi S: Feeding habits of the minke whale in the Antarctic. Rep Int Whal Comm 1979, 29:473-476.

38. Kjeld M: Concentrations of electrolytes, hormones, and other constituents in fresh postmortem blood and urine of fin whales (Balaenoptera physalus). Can J Zool 2001, 79:438-446.

39. Bester MN: The functional morphology of the kidney of the Cape fur seal, Arctocephalus pusillus (Schreber). Modoqua Ser 1975, 4:69-92.

40. Vardy PH, Bryden MM: The kidney of Leptonychotes weddelli (Pinnipedia: Phocidae) with some observations on the kidneys of two other southern phocid seals. J Morphol 1981, 167:13-34.

41. Birukawa N, Ando H, Goto M, Kanda N, Pastene LA, Urano A: Molecular cloning of urea transporters from the kidneys of baleen and toothed whales. Comp Biochem Physiol B Biochem Mol Biol 2008, 149:227-235.

42. Suzuki M: Expression and localization of aquaporin-1 on the apical membrane of enterocytes in the small intestine of bottlenose dolphins. J Comp Physiol B, Biochem Syst Environ Physiol 2010, 180:229-238.

43. Suzuki M, Endo N, Nakano Y, Kato H, Kishiro T, Asahina K: Localization of aquaporin-2, renal morphology and urine composition in the bottlenose dolphin and the Baird's beaked whale. J Comp Physiol B, Biochem Syst Environ Physiol 2008, 178:149-156.

44. Ni JY, Zhou KY: Index of smaller kidney structures of finless porpoises and urinary concentrating ability. Curr zool 1988, 34(3):243-250.

45. Fushimi K, Uchida S, Hara Y, Hirata Y, Marumo F, Sasaki S: Cloning and expression of apical membrane water channel of rat kidney collecting tubule. Nature 1993, 361:549-552.

46. Deen PM, Verdijk MA, Knoers NV, Wieringa B, Monnens $L A$, Van Os $C H$, van Oost BA: Requirement of human renal water channel aquaporin-2 for vasopressin-dependent concentration of urine. Science 1994, 264:92-95.

47. Fenton RA, Flynn A, Shodeinde A, Smith CP, Schnermann J, Knepper MA Renal phenotype of UT-A urea transporter knockout mice. J Am Soc Nephrol 2005, 16:1583-1592.

48. Ohta T: Near-neutrality in evolution of genes and gene regulation. Proc Natl Acad Sci USA 2002, 99:16134-16137.

49. Kimura M: The neutral theory of molecular evolution. Cambridge: Cambridge University Press; 1983.

50. Demetrius L: Directionality theory and the evolution of body size. Proc Natl Acad Sci U S A 2000, 267:2385-2391.

51. Alter $S E$, Rynes E, Palumbi SR: DNA evidence for historic population size and past ecosystem impacts of gray whales. PNAS 2007, 104:15162-15167.

52. Ranwez V, Delsuc F, Ranwez S, Belkhir K, Tilak M-K, Douzery EJ: OrthoMaM A database of orthologous genomic markers for placental mammal phylogenetics. BMC Evol Biol 2007, 7:241.

53. Thompson JD, Gibson TJ, Plewniak F, Jeanmougin F, Higgins DG: The CLUSTAL_X windows interface: flexible strategies for multiple sequence alignment aided by quality analysis tools. Nucl Acids Res 1997, 25:4876-4882.

54. Tamura K, Peterson D, Peterson N, Stecher G, Nei M, Kumar S: MEGA5, molecular evolutionary genetics analysis using maximum likelihood, evolutionary distance, and maximum parsimony methods. Mol Biol Evol 2011, 28:2731-2739. 
55. Yang Z: PAML 4: Phylogenetic analysis by maximum likelihood. $\mathrm{Mol}$ Biol Evol 2007, 24:1586-1591.

56. Zhou X, Xu S, Xu J, Chen B, Zhou K, Yang G: Phylogenomic analysis resolves the interordinal relationships and rapid diversification of the laurasiatherian mammals. Syst Biol 2012, 61:150-164.

57. Perelman P: Molecular phylogeny of living primates. PLOS Genet 2011, 7:e1001342.

58. Nielsen R, Yang Z: Likelihood models for detecting positively selected amino acid sites and applications to the HIV-1 envelope gene. Genetics 1998, 148:929-936.

59. Yang Z, Nielsen R: Estimating synonymous and nonsynonymous substitution rates under realistic evolutionary models. Mol Biol Evol 2000 17:32-43.

60. Yang Z, Nielsen R, Goldman N, Pedersen A-M: Codon-substitution models for heterogeneous selection pressure at amino acid sites. Genetics 2000, 155:431-449.

61. Yang Z, Wong WSW, Nielsen R: Bayes empirical bayes inference of amino acid sites under positive selection. Mol Biol Evol 2005, 22:1107-1118.

62. Zhang J: Rates of conservative and radical nonsynonymous nucleotide substitutions in mammalian nuclear genes. J Mol Evol 2000, 50:56-68.

63. Anisimova A, Yang Z: Multiple hypothesis testing to detect adaptive protein evolution affecting individual branches and sites. Mol Biol Evol 2007, 24:1219-1228.

64. Benjamini $Y$, Hochberg $Y$ : Controlling the false discovery rate: a practical and powerful approach to multiple testing. J R Stat SoC B 1995, 57:289-300.

65. Pond SLK, Frost SDW: Datamonkey, rapid detection of selective pressure on individual sites of codon alignments. Bioinformatics 2005, 21:2531-2533.

66. Zhang J, Nielsen R, Yang Z: Evaluation of an improved branch-site likelihood method for detecting positive selection at the molecular level. Mol Biol Evol 2005, 22:2472-2479.

67. Arnold K, Bordoli L, Kopp J, Schwede T: The SWISS-MODEL workspace: a web-based environment for protein structure homology modelling. Bioinformatics 2006, 22:195-201.

doi:10.1186/1471-2148-13-189

Cite this article as: Xu et al:: Adaptive evolution of the osmoregulationrelated genes in cetaceans during secondary aquatic adaptation. BMC Evolutionary Biology 2013 13:189.

\section{Submit your next manuscript to BioMed Central and take full advantage of:}

- Convenient online submission

- Thorough peer review

- No space constraints or color figure charges

- Immediate publication on acceptance

- Inclusion in PubMed, CAS, Scopus and Google Scholar

- Research which is freely available for redistribution 